Original Research Paper

\title{
Enhancing Digital Images Through Cuckoo Search Algorithm in Combination with Morphological Operation
}

\author{
${ }^{1}$ Ratna Babu, K. and ${ }^{2}$ K.V.N. Sunitha \\ ${ }^{I}$ Department of CME SUVR and SR Govt. Polytechnic for Women, Ethamukkala, Prakasam Dt., India \\ ${ }^{2}$ BVRIT Hyderabad college of Engineering for Women, Hyderabad, India
}

Article history

Received: 08-11-2013

Revised: 03-04-2014

Accepted: 23-07-2014

Corresponding Author:

Ratna Babu, K.

Department of CME SUVR and

SR Govt. Polytechnic for

Women, Ethamukkala,

Prakasam Dt., India

Email: kratnababuphd@gmail.com

\begin{abstract}
This study presents an image enhancement approach to Cuckoo Search Algorithmin with Morphological Operation. At the present time, in many image processing applications digital images are developed. Machine vision, computer interfaces, manufacturing, compression for storage and more are some of the fields of image processing application. Before using it in any applications the image has to be managed, such processing is said to be image enhancement. We propose a method to combine with an enhancing digital images through cuckoo search algorithmin and morphological operation. Therefore, the appearance of noise produces distortion in an image and thus the image will be unattractive. This decreases the discernibility of many features inside the images. In this study, we are working to overcome this drawback by getting an improved contrast value after converting the color image into grayscale image. The fundamental characteristic of this CS algorithm is that the amplitudes of its components can objectively reflect the contribution of the gray levels to the representation of image information for the best contrast value of an image. After selecting the best contrast value of an image in CS algorithm, morphological operations have to be done. In morphological operations, the intensity parameters of the image are adjusted to improve its quality. Experimental results demonstrate that the proposed approach is converted into original color image without noise and adaptive process to enhance the quality of images.
\end{abstract}

Keywords: Digital Images Enhancement, Morphological Operation, Cuckoo Search Algorithm

\section{Introduction}

Decades ago, much attention has been paid to the multi-resolution characteristic of processes and patterns in general. Good examples are image-processing, which provide different information and noise at various spatial scales. Analysts have become aware that image enhancement could be considerably improved if the scenes are viewed at multiple resolutions (Afruz et al., 2010). Basically, all satellite image-processing operations can be grouped into three categories: Image Rectification and Restoration, Enhancement and Information Extraction. Image processing with better flexibility and adaptability is highly enviable in many applications such as image transformation, correction of distortion effects, noise purge, histogram equalization and more (Krishna et al., 2010).

The objective of image enhancement is to classify and portray, as a unique grey level (or colour), the features occurring in an image in terms of the object or type of land cover these features actually represent on the ground. Normally, noise reduction is the initial process in the analysis of digital images (Nadernejad et al., 2008). Image contrast enhancement, noise elimination, thresholding, edge detection and image segmentation are the major steps involved in digital image processing techniques (Zelelew et al., 2008). The image can be affected by noise unavoidably in the process of saving and transmission and produces bad effects on the image 
processing. For eliminating such effects, it is indispensable to remove or diminish the noise and at the same time, to preserve the image information as much as possible (Singh and Kathane, 2011).

Image enhancement acting an essential role in several image processing applications, where the experts make decisions based on the image information (Al-Samaraie, 2011). To complete this task, it is imperative to increase the dynamic range of the selected features in the image, which is basically the process of image enhancement (Al-Samaraie and Majied Al Saiyd, 2011). When images change from one form to another through the processes such as imaging, scanning, or transmitting, the quality of the output image may be subordinate than that of the original input image. Thus, there is a need to enhance the quality of such images (Vij and Singh, 2011). The purpose of image enhancement is to enhance the image quality such that the processed image is superior to the original image for a certain application or set of objectives. Preprocessing the image with an enhancing filter will produce good segmentation results (Gorai and Ghosh, 2011). Image enhancement techniques are separated into two wide categories (Maini and Aggarwal, 2010):

\section{- Spatial domain methods}

- Frequency domain methods

Spatial domain enhancement method: Here, the pixel is directly gray-scale mapped to achieve an enhanced image. This method can automatically increase the entire image contrast. But, it is very sensitive to noise (Hua et al., 2011).

Frequency domain enhancement method: This method has made the best of the wavelet with the multiresolution time frequency analysis property. It has defeated the drawbacks of the wavelet transform in the image noise and has solved the sparsity problem of smooth edge target (Hafizah and Supriyanto, 2011).

In order to simplify the comparisons between the classification results for the different image enhancement techniques, the MSE classification threshold has been varied individually in each case (Preethi and Rajeswari, 2010). Image enhancement is a process of enhancing the quality of an image by improving its features. For underwater images, three significant image enhancement techniques are utilized: Contrast stretching, Histogram equalization and Contrast limited adaptive histogram equalization (Kumarrai et al., 2012). The contrast stretching process plays a vital role in improving the quality as well as the contrast of medical images (Mokhtar et al., 2009). The histogram equalization is the most common technique, used for improving the visibility of images. Suppose if an image is mostly dark, then its histogram would be skewed towards the lower end of the grey scale and all the image detail is compressed into the dark end of the histogram (Yoon et al., 2009). Two techniques such as Pixel Based Texture Synthesis and Patch Based Texture Synthesis are utilized for cleaning the gray scale image (Shah et al., 2012). Image enhancement process comprises a set of techniques, which aspire to enhance the visual appearance of an image or to convert the image to a form compatible for analysis by a human or machine. The greatest complexity in image enhancement is measuring the criterion for enrichment (Ahmed and Barskar, 2011). The objective of color enhancement can be either to augment the brightness, or to increase the saturation (Kumar and Chandrakar, 2011). Image Enhancement is one of the protruding research areas in Image Processing as it is more propitious in several applications such as Satellite Image Processing, Medical, Military, Print media and so on (Suneetha and SriKrishna, 2011).

\section{Cuckoo Search Algorithm}

Cuckoo Search Algorithmis based on the brood parasitism of some cuckoo species (Brajevic et al., 2012). In addition, CS algorithm is improved by the socalled Lévy flights, rather than by simple isotropic random walks (Layeb and Boussalia, 2012; Valian et al., 2011a). The CS was inspired by the obligate brood parasitism of some cuckoo species by laying their eggs in the nests of host birds. Some cuckoos have evolved in such a way that female parasitic cuckoos can imitate the colors and patterns of the eggs of a few chosen host species (Valian et al., 2011b). This reduces the probability of the eggs being abandoned and, therefore, increases their re-productivity. It is worth mentioning that several host birds engage direct conflict with intruding cuckoos (Yildiz, 2012; Tiwari, 2012). In this case, if host birds discover the eggs are not their own, they will either throw them away or simply abandon their nests and build new ones, elsewhere (Dhivya et al., 2011; Babukartik and Dhavachelvan, 2012). For simplicity in describing the cuckoo search, consider the following three idealized rules: (1) Each cuckoo sets one egg at a time and dump its egg in randomly chosen nest; (2) The best nests with high quality of eggs will carryover to the next generations; (3) The number of available host nests is fixed and the egg laid by a cuckoo is discovered by the host bird (Rani et al., 2012; Noghrehabadi et al., 2011). 
In this study, we enhance the image using CS algorithms and morphological operations (Ma et al. 2011). The contrast and intensity parameters of the image are adjusted by means of CS algorithm. After enhancing the input image by CS algorithm, we apply morphological operations for further enhancement of the image. The rest of the study is organized as follows: Section 3 reviews the recent research works related to the image enhancement techniques, section 4 details the steps involved in the proposed technique with necessary illustrations and mathematical formulations, section 5 discusses about the implementation results and section 6 concludes the study.

\section{Related Researches: A Review}

In this section, a handful of recent research works available in the literature are briefly reviewed. For image enhancement, (Weigel et al., 2013) have proposed a method to combine Image Inversion Microscopy (IIM) with digital holography. Furthermore, they presented a setup and some measurements as proof of principle. The Point Spread Function (PSF) of a holographic image inversion microscope was compared to the conventional PSF. They have demonstrated that the distance between the first zeros is reduced by a factor of about two. Additionally, they recorded images of 10 gratings to demonstrate the enhanced resolution and to measure a part of the optical transfer functions of the coherent, the incoherent and the image inverted case.

Lin (2011) have proposed a technique for an image enhancement approach to Infrared (IR) images for long-range sur-veillance. The IR images captured at long range usually have low contrast, low brightness and small hot objects of interest. The main characteristics of the proposed approach were that no prior knowledge aboutthe IR image was necessary and no parameters must be preset. Two main goals are sought: Adaptive contrast enhancement and enhancement of the power of high spatial frequency in IR images. In this proposed work, a novel Adaptive Histogram-Based Equalization (AHBE) was used for adaptive contrast enhancement. In this way, the proposed adaptive equalization approach can improve the enhancement effect on small hot objects embedded in an image. In addition, using a high-boost filter enhances the power of high spatial frequency in IR images and maintains the information about the original images. As a result, the diffraction effects on IR images caused by the IR optical system was ameliorated through the high-boost filter.

Zhao (2011) have presented the adaptability and effect of image enhancement, this proposed method have an image enhancement method based on Gravitational Search Algorithm (GSA), which was used for optimizing the parameters of the normalized incomplete Beta function using the characteristics of the original image, the acquired function was employed to enhance the degraded image. The simulation results show the method can effectively enhance the global contrast of the image and vision.

Zeng et al. (2012) have Many applications of histogram-based techniques for the purposes of image enhancement are wellknown. However, these techniques often fail to produce satisfactory results for a broad variety of low-contrast images. In this proposed method, they applied a new form of histogram for image contrast enhancement. The input image was first divided into several equal-sized regions according to the intensities of gradients, their corresponding statistical values of gray levels were then modified respectively and finally the processed histogram for the whole image was obtained by the summation of all the weighted values of regions. The performance of many histogram based enhancement techniques might be improved dramatically using the proposed histogram. Testing on the X-ray images validates the effectiveness of the new histogram.

Santhanam and Radhika (2010) have proposed a technique Noise identification predominant in any digital image processing algorithms, which helps in identifying the filters to smooth the image for further processing. In this proposed method, an Artificial Neural Network (ANN) based approach was proposed for noise identification. The suggested technique involved seclusion of the noise samples and extracts their statistical features, which was then applied to a neural network to identify the noise. Neural networks provided a better solution in identifying the noise.

Garg et al. (2011) have proposed Various enhancement schemes are used for enhancing an image which includes gray scale manipulation, filtering and Histogram Equalization (HE). Although these methods preserve the input brightness on the output image with a significant contrast enhancement, they may produce images with do not look as natural as the input ones. HE method was to re-map the gray levels of an image. Comparative analysis of different enhancement techniques will be carried out. This comparison was done on the basis of subjective and objective parameters. Subjective parameters were visual quality and computation time and objective parameters were Peak Signal-to-Noise Ratio (PSNR), Mean Squared Error (MSE), Normalized Absolute Error (NAE), Normalized Correlation, Error Color and Composite Peak Signal to Noise Ratio (CPSNR). 
Teymourzadeh et al. (2013) has proposed advancements in the fields of digital image processing and artificial intelligence have applied in solving many real-life problems. This could be seen in facial image recognition for security systems, identity registrations. Hence a bottleneck of identity registration is image processing. These are carried out in form of image preprocessing, image region extraction by cropping, feature extraction using Principal Component Analysis (PCA) and image compression using Discrete Cosine Transform (DCT). Hence, this research work presents a universal integration image forgery detection analysis tool with image facial recognition using Black Propagation Neural Network (BPNN) processor. The proposed designed tool is a multi-function smart tool with the novel architecture of programmable error goal and light intensity. Furthermore, its advance dual database increases the efficiency for high performance application. With the fact that, the facial image recognition will always, give a matching output or closest possible output image for every input image irrespective of the authenticity, the universal smart GUI tool is proposed and designed to perform image forgery detection with the high accuracy of $\hat{\mathrm{A}} \pm 2 \%$ error rate.

Bai et al. (2011) have proposed an infrared image enhancement algorithm through contrast enhancement. In this proposed method based on multiscale new top-hat transform. Firstly, the multiscale white and black new top-hat transforms were used to extract the multiscale light and dark infrared image regions. Then, the final light and dark infrared image regions for image enhancement are constructed by using the extracted multiscale light and dark infrared image regions. Finally, the contrast of the infrared image was enhanced through a power strategy. Experimental results on different infrared images show that the proposed algorithm could well enhance infrared image and make the possible interested targets brighter, which was very helpful for target detection and recognition.

Sridevi et al. (2012) have proposed a method digital medical powerful tools for diagnosis, treatment and surgery and plays a vital role in modern healthcare delivery. Large storage capacity is needed for storing these images and for transmitting them. This leads to the strong demand for digital medical image compression and reliable transmission. In this study, we have applied three compression methods to medical images. In all the methods discrete wavelet transform is applied followed by the corresponding compression methods. The experiments are carried on three medical images and the quality of reconstructed images is evaluated based on Compression Ratio (CR) and Peak
Signal to Noise Ratio. The results show that the SOM algorithm has higher compression ratio than FCM and FKM while maintaining the image quality and preserving the information. The results show that the SOM algorithm outperforms the existing methods FCM and FKM for medical image compression.

Achmad et al. (2011) have proposed a optical flow inter-frame enhancement techniques to improve the quality of ultrasound images by reducing the speckle noise. The performance depends on accuracy of the optical flow inter-frame reconstruction which is a part of the technique. The speckle noise of an ultrasound image forms certain pattern, of which size of the speckle varies depends on the distance from the transducer. This proposed method, that the use of warping technique prior to as well as after the optical flow to improve the accuracy of the optical flow calculation. The warping function was derived to transform the image from circular grid to rectangular grid. The technique was applied to a series of synthetic moving frames generated from actual ultrasound image taken from a patient heart. The technique improved the accuracy of motion vectors generated from the optical flow. The MSE of the reconstructed frame was also smaller using warped technique compared to that of the non-warped technique.

Rahman et al. (2011) have proposed an image enhancement and data compression methods arose from the distinct and largely separate disciplines of image processing and communications respectively, yet both are important components of current and future digital imaging systems technology. However, if image enhancement was performed before image compression, there were two issues that arise: (i) Image enhancement typically increases the contrast amount of observable detail in an image which leads to poorer compression ratios; and (ii) the radiometric information in the original data was typically irretrievably lost. In this proposed method,they address the impact of image enhancement, specifically that of the Multi-Scale Retinex with Color Restoration (MSRCR) on image compression and vice versa. They was look at the impact of compression on recovering original data from enhanced imagery given certain parameters about the enhancement process.

\section{Proposed Methodology Using Cuckoo Search Algorithmand Morphological Operation for Image Enhancement}

A set of pixels settled in rows and columns is called an image. In the evaluation and elucidation of image data, image processing plays a dynamic role. Image 
enhancement is an austere and attractive field of image processing. In this study, in association with the morphological operation we applied cuckoo search algorithm. Firstly, to regulate the intensity and contrast parameters of the input image the cuckoo search algorithm is used. Then morphological operations are performed for the improvement of the image and lastly, a noise free image is attained. In detail the process is explained below.

Let $I$ be an image of dimension $\mathrm{P} \times \mathrm{Q}$ and by our hybrid technique it is to be enhanced. The image $I$ which is in RGB color space is first converted into grayscale color space. The following Equation 1 signifies the transformation process:

$$
I_{g y}=0.289 * r+0.5870 * g+0.1140 * b
$$

Now, $R, G, B$ are the components of a RGB single image and $I_{g r}$ is the converted grayscale image. The mean of the transformed grayscale image $I_{g r}$ is calculated and it is used in the following process. The formula for calculating the mean $\mu$ of the image is given below Equation 2:

$$
\mu=\frac{\sum_{p=0}^{p-1} \sum_{q=0}^{Q-1} I p q}{P \times Q}
$$

The image is then applied to the Cuckoo Search Algorithm to get an enhanced image and the process that takes place in Cuckoo Search Algorithm is described briefly in the below section.

Image Enhancement Based on Cuckoo Search Algorithm.

Cuckoo Search (CS) is an optimization algorithm. Each egg in a nest represents a solution and a cuckoo egg represents a new solution. This algorithm is inspired by the aggressive reproductive strategy of somecuckoo species such as the Ani and Guira cuckoos. The aim is to use the new and potentially better solutions (cuckoos) to replace a not-so-good solution in the nests. These cuckoos lay their eggs in communal nests, though they may remove others' eggs to increase the hatching probability of their own eggs. Quite a number of species engage the obligate brood parasitism by laying their eggs in the nests of other host birds. This algorithm works with three basic principles. First principle: Each cuckoo lays one egg at a time and dumps its egg in a randomly chosen nest. Second principle: The best nests with high quality of eggs will carry over to the next generation and third principle: The number of available host nests is fixed and the egg laid by a cuckoo is discovered by the host bird with a probability $P_{a} \in[0,1]$. In this case, the host bird can either get rid of the egg, or simply abandon the nest and build a completely new nest.

The third assumptions can be approximated as the fraction $\mathrm{p}$ of the ' $n$ ' nests is replaced by new nests (new random solutions). Many studies have shown that the flight behavior of many animals and insects has demonstrated the typical characteristics of Lévy flights. Lévy flight is defined as a random walk with the steplengths based on a heavy-tailed probability distribution. Consequently, such behavior has been emulated to optimization and global optimal search with a promising capability.

In this study, the standard CS algorithm has been used to enhance its performance. In this case, when generating new solutions $x(t+1)$ for a cuckoo $\mathrm{i}$, a Levy flight integrating with controls the search ability is performed Equation 3:

$x_{i}^{t+1}=x_{i}^{t}+\alpha \oplus \operatorname{Le} v y(\lambda)$

where, $a>0$ is the step size, which should be related to the scale of the problem of interest. The product $\oplus$ means entry-wise multiplications. In this research work, we consider a Lévy flight in which the steplengths are distributed according to the following probability distribution Equation 4:

$L e^{\prime} v y \lambda=t^{-\lambda},(1<\lambda \leq 3)$

Which has an infinite variance here, the consecutive jumps/steps of a cuckoo essentially form a random walk process which obeys a power-law steplength distribution with a heavy tail. It is worth pointing out that, in the real world, if a cuckoo's egg is very similar to a host's eggs, then this cuckoo's egg is less likely to be discovered, thus the fitness should be related to the difference in solutions. Therefore, it is a good idea to do a random walk in a biased way with some random step sizes.

The above Fig. 1 Represents the entire process of image enhancement using CS algorithms and Morphological operations. By using CS algorithms and Morphological operations, the intensity parameters are adjusted and an enhanced output image is obtained. However, in our proposed method cuckoo search, we obtained a better solution. Then, morphological operations are performed for improving the image by adjusting the intensity parameters. The morphological operations are explained below. 


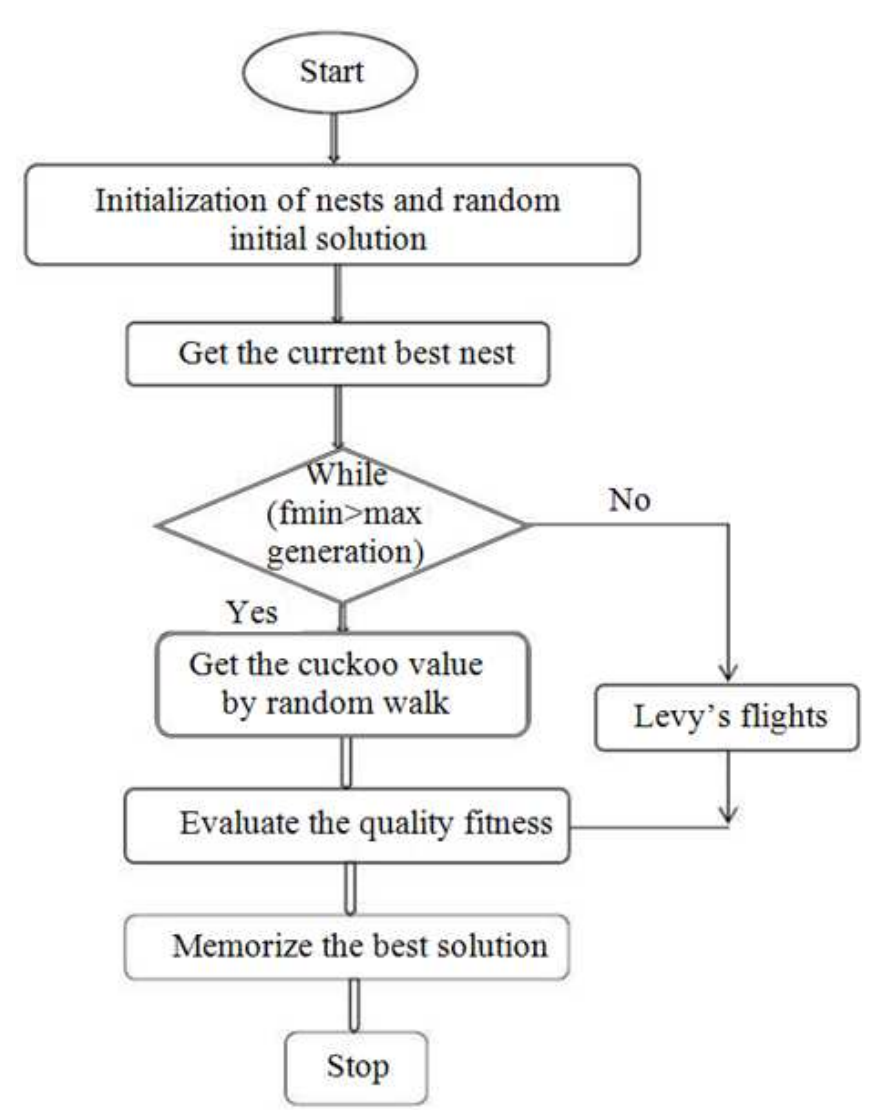

Fig. 1. Proposed CS algorithm for Image enhancement

\section{Morphological Operations}

In this section, the contrast value of the image is improved by using the morphological operations. Here, the dilation process is performed by placing the constructing element of the image and descending it transversely to the image in a routine related to complexity. The output image is acquired by utilizing a structuring element in the input image. The value of each pixel in the output image is constructed by measuring the conformed pixels in the input image. By adjusting the contrast and intensity parameters, the image $I$ is converted into binary form $I_{b}$. Then, by applying the Equation 5, an enhanced image is obtained through the morphological operation that utilizes the structuring element 'se':

$$
I_{b} \oplus S e=S e \oplus I_{b}
$$

\section{Results}

In the working platform of MATLAB (version 7.11), the performance of the proposed image enhancement is applied using a CS algorithm and morphological operation. The image enhancement process is tested with different images and the upcoming value have shown the performance of the proposed work. Primarily, by the cuckoo search algorithm the image is processed and then for improving the image, the morphological operation is implemented. Lastly, an enhanced image is attained. In discussion, in order to adjust the contrast value a CS algorithm is initially applied to the input images. It has been assessed until it selects an optimal contrast value. Later, morphological operation 'Imdilate' is applied.

Figure 2-6 have shown the original images and its enhanced images version of our work. Table 1 describes the performance of our proposed CS algorithm work and previous existing works like GA, PSO, ABC respectively. In Fig. 7 shows the graphical representation of the comparison result. Here, our proposed work get 17.627926 and exitsting value give $16.0594,17.113572,17.334228$ respectally and the performance show that the gloomy images are effectively improved with image enhancement technique using CS algorithms and morphological operations. 


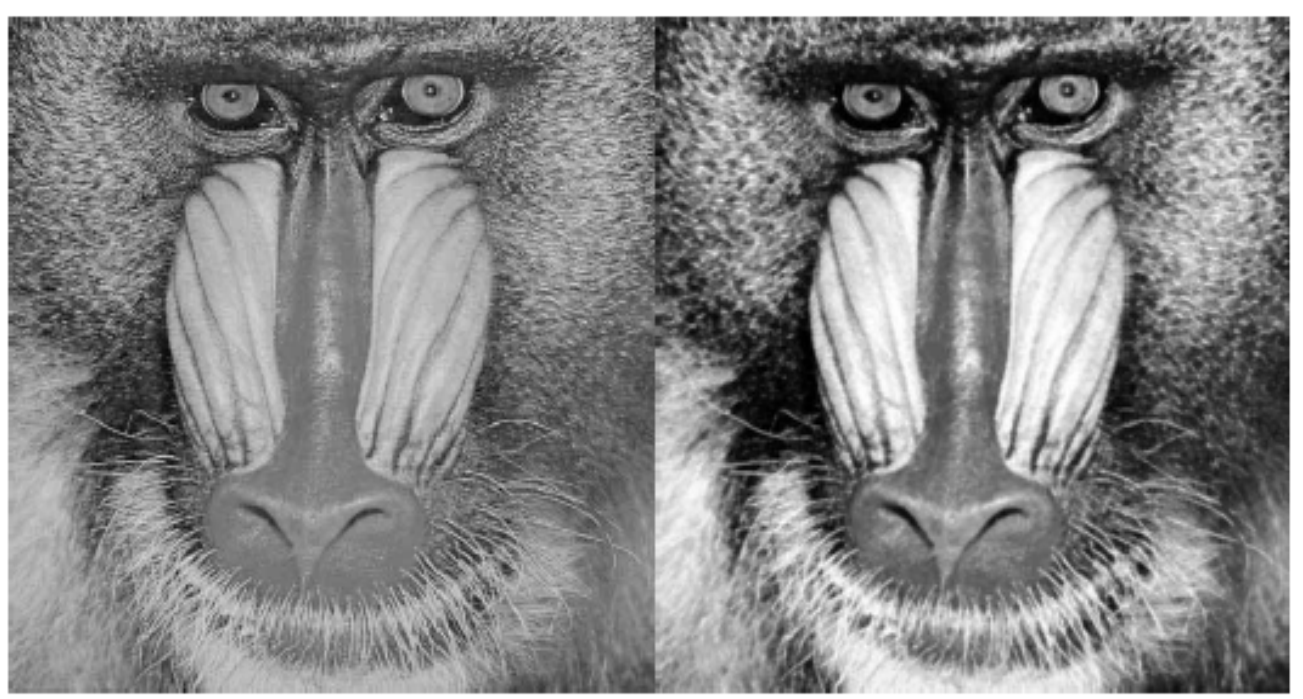

Fig. 2. Original baboon image and its enhanced image

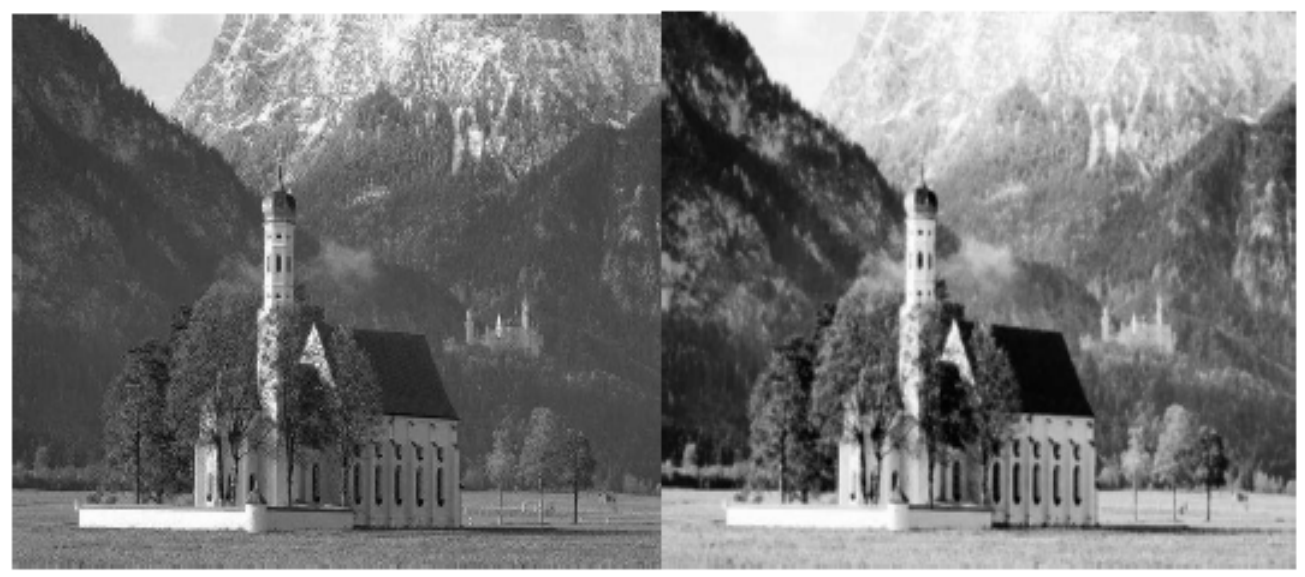

Fig. 3. Original scenery image and its enhanced image
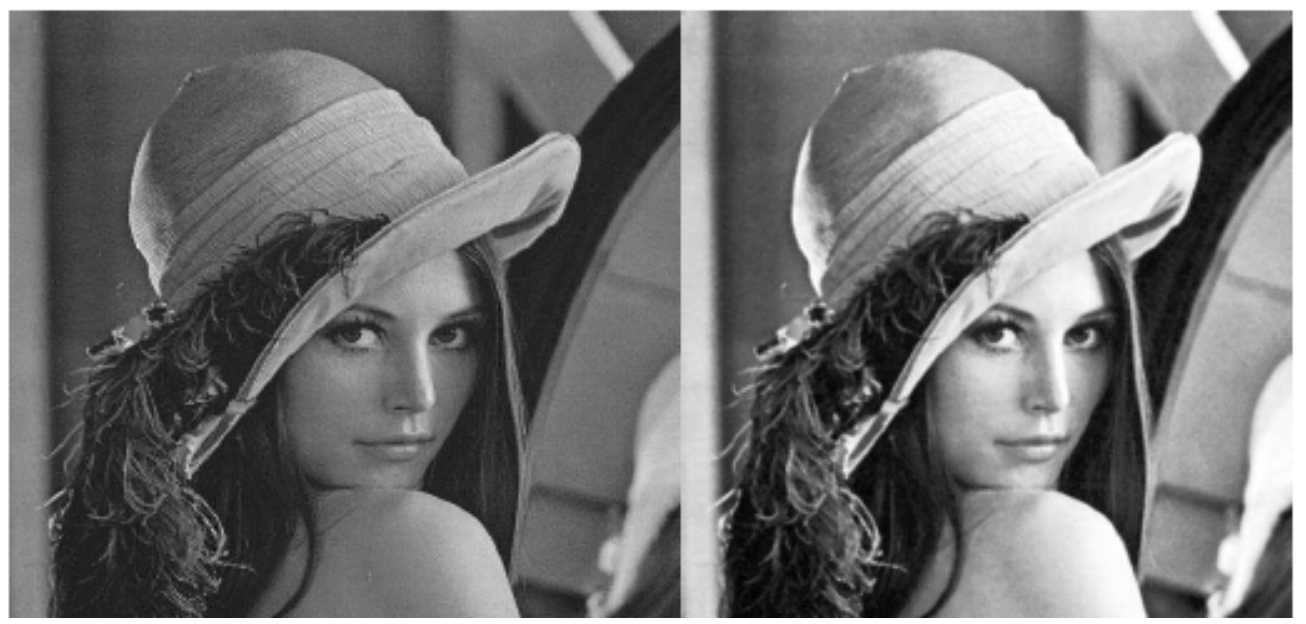

Fig. 4. Original Lena image and its enhanced image 


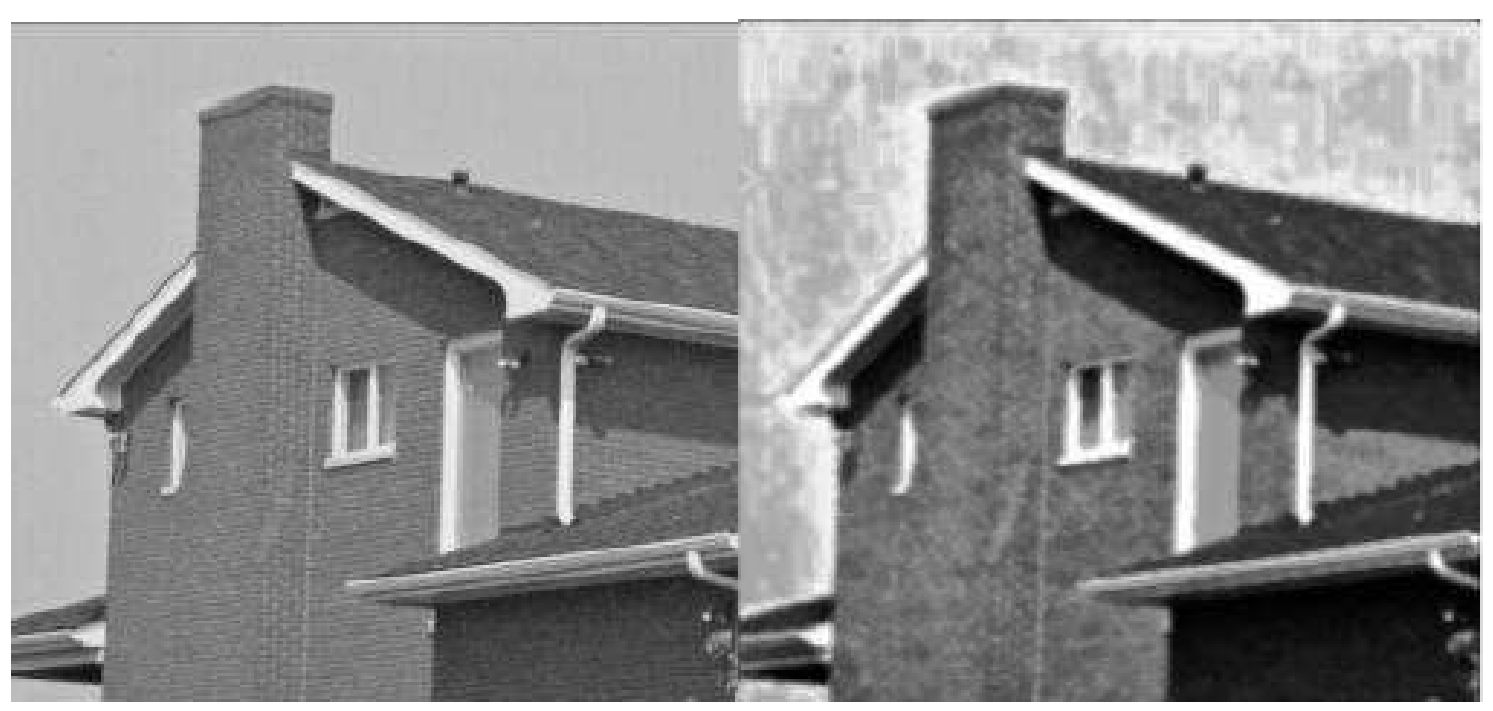

Fig. 5. Original House image and its enhanced image

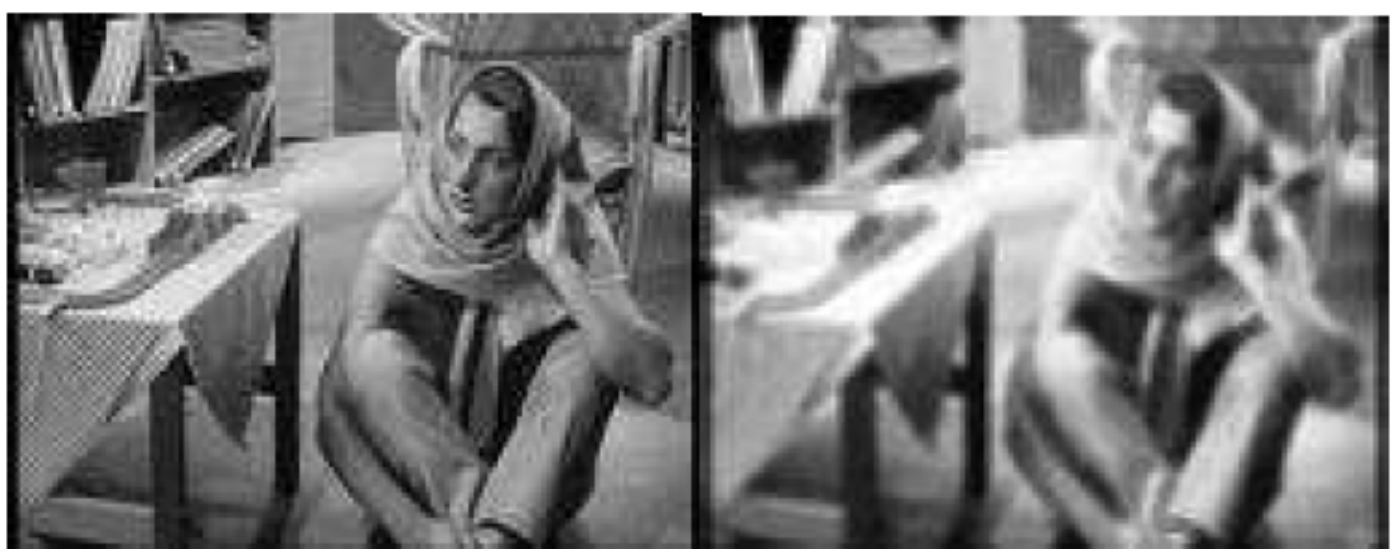

Fig. 6. Original Man image and its enhanced image

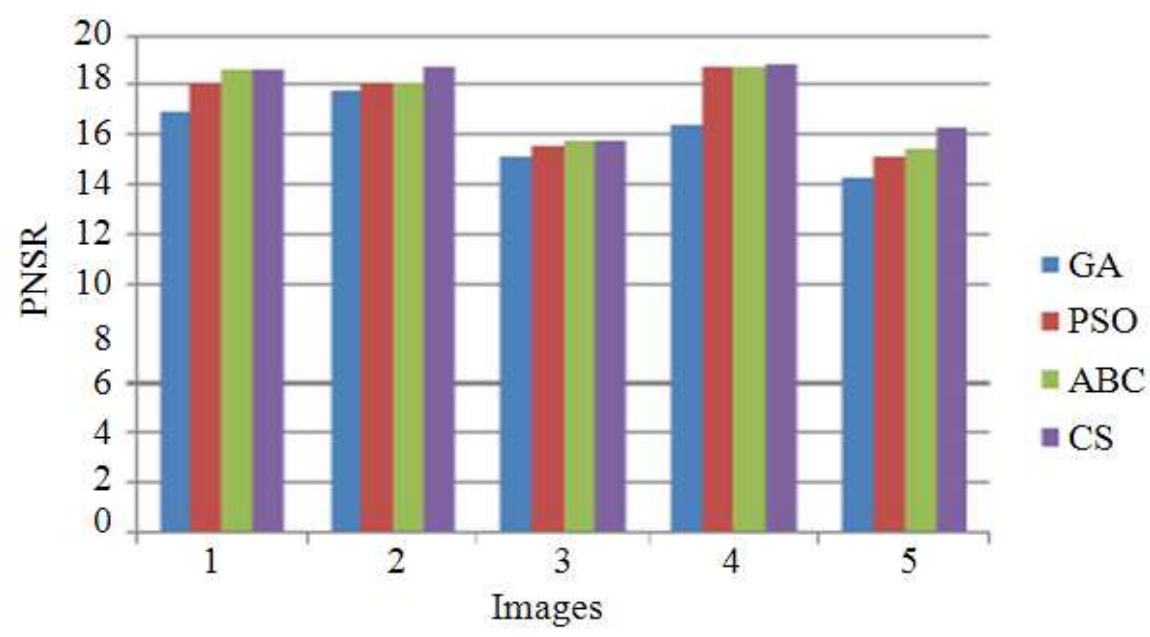

Fig. 7. Comparison of proposed work with our previous works 
Table 1. Comparison of PSNR values of the proposed work and the previous work

\begin{tabular}{llll}
\hline GA (Previous work) & PSO (Previous work) & ABC (Previous work) & CS (Proposed work) \\
\hline 16.8841 & 18.11698 & 18.65656 & 18.61738 \\
17.7551 & 18.06440 & 18.07211 & 18.71658 \\
15.0671 & 15.56377 & 15.76718 & 15.71828 \\
16.3827 & 18.71612 & 18.76172 & 18.85339 \\
14.208 & 15.10659 & 15.41357 & 16.23400 \\
\hline
\end{tabular}

\section{Discussion}

Image enhancement algorithms offer a wide variety of approaches for modifying images to achieve visually acceptable images. The choice of such techniques is a function of the specific task, image content, observer characteristics, and viewing conditions. The point processing methods are most primitive, yet essential image processing operations are used primarily for contrast enhancement.

By observing of this image enhancement by using a CS algorithm and morphological operation. The image enhancement process is implemented with different images and the upcoming value have shown the performance. In Fig. 2-6, have to used input image and output result with compare all previoue result and then for improving the image, the morphological operation is implemented.

Digital observasions were done by matching the reconstructed frames in Fig. 2 to 6 . Digitally, the dewarped enhanced result is closer to the original image compare to the enhanced image. It can be seen from Table 1, that the Image enhancement algorithms gives smaller PSNR value, hence we can conclude that this technique perform better than the original technique.

\section{Conclusion}

We have presented an image enhancement technique using CS algorithms and morphological operations in the digital world. In the beginning, the original color image was converted into grayscale image and then, the contrast value of the image was attained by calculating the fitness through CS algorithm. Later for further improvement, in order to improve the quality of the image the best contrast value of the image was selected and morphological operations were done by adjusting the intensity parameters. Finally, the grayimage was again converted into original color image without noise. Similarly, with the previous three works the proposed work was compared and it has proved to be more precise. The keeping of the parameters of our discusstion and approach to improving the image enhancement technique. Using MATLAB 7.11, the proposed approach was applied and its performance was valued. In relation to the simulation results, in terms of the accuracy of the solutions found the proposed approach performed well in a number of benchmark problems.

\section{Author's Contributions}

All authors equally contributed in this work.

\section{Ethics}

This article is original and contains unpublished material. The corresponding author confirms that all of the other authors have read and approved the manuscript and no ethical issues involved.

\section{References}

Afruz, J., V. Wilson and S.E. Umbaugh, 2010. Frequency domain pseudo-color to enhance ultrasound images. Comput. Inform. Sci., 3:24-34.

Ahmed, G.F. and R. Barskar, 2011. A study on different image retrieval techniques in image processing. Int. J. Soft Comput. Eng., 1: 247-251.

Al-Samaraie, M.F. and N.A. Majied Al Saiyd, 2011. Medical colored image enhancement using wavelet transform followed by image sharpening. Proc. Ubiquitous Comput. Commun. J., 6: 1-8.

Achmad, B., M.M. Mustafa and A. Hussain, 2011. Warped optical-flow inter-frame reconstruction for ultrasound image enhancement. J. Comput. Sci., 7: 1532-1540, DOI: 10.3844/jcssp.2011.1532.1540

Babukartik, R.G. and P. Dhavachelvan, 2012. Hybrid Algorithm using the advantage of ACO and Cuckoo Search for Job Scheduling. Int. J. Inform. Technol. Convergence Services, 2: 25-34. DOI: $10.5121 /$ ijitcs. 2012.2403

Bai, X., F. Zhou and B. Xue, 2011. Infrared image enhancement through contrast enhancement by using multiscalenew top-hat transform. Infrared Phys. Technol., 54: 61-69. DOI: $10.1016 /$ j.infrared.2010.12.001

Brajevic, I., M. Tuba and N. Bacanin, 2012. Multilevel image thresholding selection basedon the cuckoo search algorithm. Proceedings of the Advances in Sensors, Signals, Visualization, Imaging and Simulation, (VIS’ 12), pp: 217-222. 
Dhivya, M., M. Sundarambal and L.N. Anand, 2011. Energy efficient computation of data fusion in wireless sensor networks using Cuckoo Based Particle Approach (CBPA). Int. J. Commun., Netw. Syst. Sci., 4: 249-255. DOI: $10.4236 /$ ijens.2011.44030

Garg, R., B. Mittal and S. Garg, 2011. Histogram equalization techniques for image enhancement. Int. J. Electron. Commun. Technol., 2: 107-111.

Gorai, A. and A. Ghosh, 2011. Hue-preserving color image enhancement using particle swarm optimization. Proceedindgs of the IEEE Recent Advances in Intelligent Computational Systems, Sept. 22-24, IEEE Xplore Press, Trivandrum, pp: 563-568. DOI: 10.1109/RAICS.2011.6069375

Hafizah, M.W. and E. Supriyanto, 2011. Comparative evaluation of ultrasound kidney image enhancement techniques. Int. J. Comput. Applic., 21: 15-19. DOI: $10.5120 / 2524-3432$

Hua, Z., Y. Li and J. Li, 2011. Image nonlinear enhancement algorithm based on nonsubsampled contourlet transform. Int. J. Digital Content Technol. Applic., 5: 43-51.

Krishna, K.S.R., A.G. Reddy, M.N.G. Prasad, K.C. Rao and M. Madhavi, 2010. Genetic algorithm processor for image noise filtering using evolvable hardware. Int. J. Image Proc., 4: 240-251.

Kumar, M. and C. Chandrakar, 2011. An Overview of Image enhancement techniques. Int. J. Technol., 1: $15-19$.

Kumarrai, R., P. Gour and B. Singh, 2012. Underwater image segmentation using clahe enhancement and thresholding. Int. J. Emerg. Technol. Adv. Eng., 2: 118-123.

Layeb, A. and S.R. Boussalia, 2012. A novel quantum inspired cuckoo search algorithm for bin packing problem. Int. J. Inform. Technol. Comput. Sci., 4: 58-67. DOI: $10.5815 / \mathrm{ijitcs}$.2012.05.08

Lin, C.L., 2011. An approach to adaptive infrared image enhancement for long-range surveillance. Infrared Phys. Technol., 54: 84-91. DOI: 10.1016/j.infrared.2011.01.001

Ma, M., J. Liang, M. Guo, Y. Fan and Y. Yin, 2011. SAR image segmentation based on Artificial Bee Colony algorithm. Applied Soft Comput., 11: 52055214. DOI: 10.1016/j.asoc.2011.05.039

Maini, R. and H. Aggarwal, 2010. A comprehensive review of image enhancement techniques. J. Comput., 2: 8-13.

Valian, E., S. Mohanna and S. Tavakoli, 2011a. Improved cuckoo search algorithglobal optimization. Int. J. Commun. Inform. Technol., 1: 31-44.

Valian, E., S. Mohanna and S. Tavakoli, 2011b. Improved cuckoo search algorithm for feedforward neural network training. Int. J. Artificial Int. Applic., 2: 36-43. DOI: 10.5121/ijaia.2011.2304
Mokhtar, N.R., N. Hazlyna, M.Y. Mashor, H. Roseline and N.F. Mohd et al., 2009. Image enhancement techniques using local, global, bright, dark and partial contrast stretching for acute leukemia images. World Congress Eng., 1: 807-807.

Nadernejad, E., H. Koohi and H. Hassanpour, 2008. PDEs-based method for image enhancement. Applied Math. Sci., 2: 981-993.

Rani, K.N.A., M.F.A. Malek and S.C. Neoh, 2012. Nature-inspired cuckoo search algorithm for side lobe suppression in a symmetric linear antenna array. Radioengineering, 21: 865-874.

Noghrehabadi, A., M. Ghalambaz, M. Ghalambaz and A. Vosough, 2011. A hybrid power series-cuckoo search optimization algorithm to electrostatic deflection of micro fixed-fixed actuators. Int. J. Multidisciplinary Sci. Eng., 2: 22-26.

Preethi, S.J. and K. Rajeswari, 2010. Image enhancement techniques for improving the quality of colour and gray scale medical images. Int. J. Comput. Sci. Eng., 1: 18-23.

Rahman, Z., D.J. Jobson and G.A. Woodell, 2011. Investigating the relationship between image enhancement and image compressionin the context of the multi-scale retinex. J. Visual Commun. Image Represent., 22: 237-250. DOI: $10.1016 /$ j.jvcir.2010.12.006

Shah, A., P. Bhatt and K.J. Modi, 2012. Image enhancement techniques by texture synthesis. Int. J. Emerg. Technol. Adv. Eng., 2: 97-101.

Singh, S.K. and A. Kathane, 2011. Various methods for edge detection in digital image processing. Int. J. Comput. Sci. Technol., 2: 188-190.

Suneetha, A. and A. SriKrishna, 2011. A new method of image enhancement in spatial domain using histogram equalization, smoothing and fuzzy technique. Int. J. Comput. Sci. Technol., 2: 77-79.

Santhanam, T. and S. Radhika, 2010. A novel approach to classify noises in images using artificial neural network. J. Comput. Sci., 6: 506-510. DOI: $10.3844 /$ jcssp.2010.506.510.

Sridevi, S., V.R. Vijayakumar and V.S. JebaKumari, 2012. Self organizing map cluster approach for wavelet based medical image compression. Am. J. Eng. Applied Sci., 5: 275-281. DOI: 10.3844/ajeassp.2012.275.281

Tiwari, V., 2012. Face recognition based on cuckoo search algorithm. Indian J. Comput. Sci. Eng., 3: 401-405.

Teymourzadeh, R., A.A. Laadi, Y.S. Algnabi, M.D.S. Islam and S.H.M. Ali et al., 2013. Design an advance computer-aided tool for image authentication and classification. Am. J. Applied Sci., 10: 696-705. DOI: 10.3844/ajassp.2013.696.705 
Vij, K. and Y. Singh, 2011. Comparison between different techniques of image enhancement. Int. J. VLSI Signal Proc. Appli., 1: 112-117.

Weigel, D., T. Elsmann, H. Babovsky, A. Kiessling and R. Kowarschik, 2013. Combination of the resolution enhancing image inversion microscopywith digital holography. Optics Commun., 291: 110-115. DOI: 10.1016/j.optcom.2012.10.072

Yildiz, A.R., 2012. Cuckoo search algorithm for the selection of optimal machining parameters in milling operations. Int. J. Adv. Manufactur. Technol., 64: 5561. DOI: $10.1007 / \mathrm{s} 00170-012-4013-7$

Yoon, H., Y. Han and H. Hahn, 2009. Image contrast enhancement based sub-histogram equalization technique without over-equalization noise. Proc. World Acad. Sci. Eng. Technol., 50: 176-182.
Zelelew, H.M., A.T. Papagiannakis and E. Masad, 2008. Application of digital image processing techniques for asphalt concrete mixture images. Proceedings of the International Association for Computer Methods and Advances in Geomechanics, (MAG' 08), Mill for Modified Bitumen, pp: 119-124.

Zeng, M., Y. Li, Q. Menga, T. Yang and J. Liu, 2012. Improving histogram-based image contrast enhancement using gray-level information histogram with application to X-ray images. Optik Int. J. Light Electron Opt., 123: 511-520. DOI: $10.1016 /$ j.ijleo.2011.05.017

Zhao, W., 2011. Adaptive image enhancement based on gravitational search algorithm. Procedia Eng., 15: 3288-3292. DOI: 10.1016/j.proeng.2011.08.617 